\title{
Forensic Biomechanics/Functional Anatomy/Physiology Analysis of Erectile Dysfunction: A Defense Perspective in a Product Liability Case
}

\section{Patrick Hannon}

Emeritus Faculty, Northern Arizona University, Department of Biology, USA

*Corresponding author: Patrick Hannon, Department of Biology, Northern Arizona University, USA, Tel: 480-816-0930; E-mail: Hannon@hannonbiomechanics.com

Received date: January 25, 2016; Accepted date: January 27, 2016; Published date: February 10, 2016

Copyright: ( 2016 Hannon P. This is an open-access article distributed under the terms of the Creative Commons Attribution License, which permits unrestricted use, distribution and reproduction in any medium, provided the original author and source are credited.

\section{Editorial}

In forensic biomechanics, most of us who provide expertise in this discipline have a large percentage of vehicle accident cases [1]. Certainly, as experts we appreciate unusual cases and new challenges outside the box of auto accidents. This specific case involved the defense of a bicycle seat manufacturer in a claim involving a middle aged man who reported that after an approximate 30 mile ride, the nose of his bicycle seat saddle had deformed during the last few miles of his ride resulting in his chronic erectile dysfunction (i.e. ED).

Some weeks later, the plaintiff did see an urologist. However, only patient self-report measures determined the ED diagnosis without any objective or subjective testing to confirm the claimed condition. The legal compensation claims of this man included loss of consortium and finally the loss of his marriage resulting from his ED.

The erectile dysfunction medical and biomechanical literature does address serious acute trauma to the penis, perineum or pelvis resulting from falls, motorcycle and automobile accidents, penis fracture during intercourse and during fracture fixation of the lower extremities on an orthopedic fracture table. Furthermore, acute trauma resulting from direct impact to the perineum does have the potential to produce male erectile dysfunction. One 1995 research effort based on a $91 / 2$ year longitudinal study of a sample of 131 men based upon a much larger population found that persistent changes in erectile function followed blunt pelvic or blunt perineum trauma. An estimate of some 600,000 American men are afflicted by traumatic vasculogenic impotence with some 250,000 of this population segment afflicted by sports injuries which involve blunt impact loading to the perineum or pelvis.

A further review of the literature indicated that the compressive stresses in the perineal area may produce erectile dysfunction in men with prolonged bicycle riding. Penile erection requires adequate arterial flow into the penis, a viable venocculsive mechanism, relaxation of the lacunar musculature of the corpora cavernosa and physiologically intact pudendal and cavernous nerves (nerves that course the perineal area). Studies indicate an occasional problem in serious bicyclists who have lost the ability to attain an erection after repeated multi-day rides. Treatment consists of eliminating the activity and placing additional padding within the bicycling shorts and/or using additional padding on the saddle seat of the bicycle.

Finally, an experimental study is reported by Nayal et al. (1999) involving measures of penis glans oximetry (transcutaneous penile oxygen partial pressure) in 25 healthy males (repeated measures study design) [2]. The measurements were taken during: 1) standing off of the bicycle, 2) seated and cycling on the stationary bicycle (two separated trials for each subject) and 3) cycling in a standing position on the bicycle. Mean partial pressure of oxygen (due to arterial blood perfusion of the penis glans) indicated normal values while standing (mean $\mathrm{pO}_{2}=61.4$ millimeters of mercury) or standing cycling (mean $\mathrm{pO}_{2}=68$ millimeters of mercury). However, cycling in a seated position produced means of only $\mathrm{pO}_{2}=19.4$ millimeters of mercury on the first seated trial and $\mathrm{pO}_{2}=18.4$ millimeters of mercury on the second seated trial. Clearly, vascular perfusion was significantly (but temporarily) decreased during the seated cycling position. The authors reported a return to normal penile perfusion $\mathrm{pO}_{2}$ levels within 10 minutes after seated bicycling.

My approach in this matter was to perform testing on a number of different bicycling seats that were provided to me by the manufacturer. In a single blind study, one cyclist subject sat on 7 different seats while pedaling on a stationary bicycle. We wrapped the top of each seat including the front seat nose with a compressive stress sensitive material (Novel EMED System-Germany; Pedar insole system). The results provided us with force distribution quantitative measures along with three dimensional figures of the compressive stresses in Newtons $/ \mathrm{cm}^{2}$. The important result was that the actual damaged seat and a same make and model exemplar seat both outperformed all of the other five seats in terms of reducing the compressive stresses within the perineum of our exemplar subject. This result coupled with the fact that the literature does not support a relationship between 1) a one exposure 30 mile bike ride and 2) acute or chronic ED, led to my conclusion that the bicycle seat (deformed or not) was not the etiology of the plaintiff's ED in spite of plaintiff's reported temporal correctness.

Therefore, in spite of findings that report and support erectile dysfunction from prolonged and repeated exposure to bicycling in the literature, there was no such support for the plaintiff's claim in this litigation. These findings once again emphasize that when two events simply occur within a relatively short time; what must follow is a biomechanics "Injury Causation Analysis" in order to evaluate causation. Furthermore, this case illustrates the problems with medical conditions that are not documented by solid objective testing when such testing is feasible from a medical/technical perspective.

\section{References}

1. Hannon P, in Hannon and Knapp (2006-2008), Forensic Biomechanics, Lawyers and Judges Pub, Tucson, Az 151-172.

2. Nayal W, Schwarzer U, Klotz, T, Heidenreich, A. Engelmann U, et al. (1999) Transcutaneous penile oxygen pressure during bicycling. British Journal of Urology International 83: 623-625. 